\title{
Demonstration of the hadron mass origin from the QCD trace anomaly
}

\author{
Fangcheng He, ${ }^{1, *}$ Peng Sun $\oplus^{2, \dagger}$ and Yi-Bo Yang ${ }^{1,3,4,5, \hbar}$ \\ $(\chi \mathrm{QCD}$ Collaboration) \\ ${ }^{1}$ CAS Key Laboratory of Theoretical Physics, Institute of Theoretical Physics, \\ Chinese Academy of Sciences, Beijing 100190, China \\ ${ }^{2}$ Nanjing Normal University, Nanjing, Jiangsu, 210023, China \\ ${ }^{3}$ School of Fundamental Physics and Mathematical Sciences, Hangzhou Institute for Advanced Study, \\ UCAS, Hangzhou 310024, China \\ ${ }^{4}$ International Centre for Theoretical Physics Asia-Pacific, Beijing/Hangzhou, China \\ ${ }^{5}$ School of Physical Sciences, University of Chinese Academy of Sciences, Beijing 100049, China
}

(Received 30 January 2021; accepted 15 September 2021; published 7 October 2021)

\begin{abstract}
Quantum chromodynamics (QCD) claims that the major source of the nucleon invariant mass is not the Higgs mechanism but the trace anomaly in the QCD energy-momentum tensor. Although experimental and theoretical results support such a conclusion, a direct demonstration is still absent. We present the first lattice QCD calculation of the quark and gluon trace anomaly contributions to the hadron masses, using the overlap fermion on the $2+1$-flavor dynamical domain wall quark ensemble at $m_{\pi}=340 \mathrm{MeV}$ and lattice spacing $a=0.1105 \mathrm{fm}$. The result shows that the gluon trace anomaly contributes to most of the nucleon mass, the contribution in the pion state is smaller than that in others nearly by a factor of $\sim 10$, and the gluon trace anomaly density in the center of the pion is negative. The gluon trace anomaly coefficient $\beta / g^{3}=-0.056(6)$ we obtained is consistent with its regularization-independent leading-order value $\left(-11+\frac{2 N_{f}}{3}\right) /(4 \pi)^{2}$ perfectly.
\end{abstract}

DOI: $10.1103 /$ PhysRevD.104.074507

\section{INTRODUCTION}

The most essential and nontrivial feature of the quantum field theory is the regularization. It introduces a tiny and artificial modification on the short-distance part of the original theory to make the contributions from the virtual particle loops finite and calculable. Regularization efforts can be absorbed into the renormalization of fields and the coupling constants, and are then irrelevant to the longdistance physics. Thus, the exceptional cases are called "quantum anomalies."

Taking the trace anomaly of the QCD energy-momentum tensor (EMT) with quark field $\psi$ and gluon field strength

\footnotetext{
* Corresponding author. hefc123@itp.ac.cn

Corresponding author. sunpeng@njnu.edu.cn

* Corresponding author. ybyang@itp.ac.cn

Published by the American Physical Society under the terms of the Creative Commons Attribution 4.0 International license. Further distribution of this work must maintain attribution to the author(s) and the published article's title, journal citation, and DOI. Funded by SCOAP.
}

tensor $F_{\mu \nu}$ as an example, the gluon part of the original EMT is traceless, but it is unavoidable to introduce a nonvanishing trace term on the EMT during the regularization, like the dimensional regularization case discussed in Ref. [1]. In lattice regularization, the renormalization eliminates the regularization dependence in the additional trace terms and converts them into something proportional to the strong coupling constant $[2,3]$. The renormalized EMT trace can be expressed as

$$
T_{\mu}^{\mu}=H_{m}+\left(\gamma_{m} H_{m}+\frac{\beta}{2 g} F^{2}\right),
$$

where the quark mass term $H_{m}=\sum_{q} m_{q} \bar{q} q$ is the classical trace of EMT, and both the other terms are the trace anomaly. The anomalous terms are proportional to the anomalous dimension of the quark mass $m_{q}, \gamma_{m}=-\frac{\mu}{m} \frac{\partial m}{\partial \mu}=$ $\frac{2}{\pi} \alpha_{s}+\mathcal{O}\left(\alpha_{s}^{2}\right)$, and to that of the strong coupling $\alpha_{s}=\frac{g^{2}}{4 \pi}$, $\frac{\beta}{2 g}=\frac{\mu^{2}}{2 \alpha_{s}} \frac{\partial \alpha_{s}}{\partial \mu^{2}}=\left(-\frac{11}{8 \pi}+\frac{N_{f}}{12 \pi}\right) \alpha_{s}+\mathcal{O}\left(\alpha_{s}^{2}\right)$, respectively.

The trace anomaly leads to the most nontrivial feature of QCD: quantum particles like nucleons can have positive masses, even though the light quark mass is small and the gluon is massless. The argument is simple: the hadron mass can be expressed as [4-8] 


$$
M_{H}=\left\langle T_{\mu}^{\mu}\right\rangle_{H}=\left(1+\gamma_{m}\right)\left\langle H_{m}\right\rangle_{H}+\frac{\beta}{2 g}\left\langle F^{2}\right\rangle_{H},
$$

with $\langle O\rangle_{H} \equiv\langle H|O| H\rangle$. Note that one needs to subtract the vacuum expectation value of $F^{2}$ in the calculation of the hadron matrix element $\left\langle F^{2}\right\rangle_{H}$. The gluon trace anomaly $\frac{\beta}{2 g}\left\langle F^{2}\right\rangle_{H}$ can contribute a positive hadron mass even in the chiral limit where $m_{q} \rightarrow 0$ and $\left\langle H_{m}\right\rangle_{H}$ vanish. The only exception is the pion mass: its mass will be zero in the chiral limit, and then the contribution of the trace anomaly will approach zero. Recently, there have been some studies aiming to understand the QED trace anomaly effect in the Lamb shift $[8,9]$.

The above argument can be verified indirectly through the sum rule in Eq. (2): The nucleon mass $M_{N}$ can be measured at $10^{-10}$ accuracy, and the $\left\langle H_{m}\right\rangle_{H}$ values from the three light flavors can be extracted from the $\mathrm{SU}(3)$ flavor breaking of the baryons and/or explicit lattice QCD calculations, which arrive at a value of about $90 \mathrm{MeV}$ $[10,11]$. The heavy quark contribution is canceled by the flavor dependence of $\frac{\beta}{2 g}$ at the leading order based on the heavy quark approximation $[6,12]$ :

$$
m_{Q} \bar{Q} Q_{\overrightarrow{m_{Q} \rightarrow \infty}}-\frac{\alpha_{s}}{12 \pi} F^{2}+\mathcal{O}\left(\alpha_{s}^{3}\right),
$$

and then it is effectively decoupled.

In terms of Eq. (3), the heavy quark contribution in $\left\langle H_{m}\right\rangle_{H}$ terms is canceled by the heavy quark flavor dependence in $\frac{\beta}{2 g}$ at the leading order in Eq. (2).

Most of the nucleon mass is contributed by the second term on the right-hand side of Eq. (2), while only the light quark flavor dependence remains in $\frac{\beta}{2 g}$ at the leading order. The asymptotic freedom and confinement of QCD require the QCD coupling to be stronger at larger distances, and then $\beta$ is negative; thus, the expectation value of $F^{2}$ in the nucleon $\left\langle F^{2}\right\rangle_{N}$ should be negative.

The experimental measurement of the trace anomaly arouses great interest and is considered one of the major scientific goals of the future Electron-Ion Collider (EIC) [13] and EicC [14]. One approach to detecting the trace anomaly is measuring the cross section of exclusive photoproduction of heavy quarkonium, which depends on the gluon condensate at the nonrelativistic limit [15-17]. The gluon gravitational form factors can relate to $J / \Psi$ production amplitude in the large momentum limit, which is also a possibility worth further investigation [18]. There are also other theoretical discussions on the related experimental possibility $[19,20]$.

On the theoretical side, the central challenge is verifying whether the difference between a hadron mass and its quark mass contribution actually comes from the trace anomaly effect. Such a calculation is highly nonperturbative and can only be performed by lattice QCD, but the chiral symmetry breaking in the quark mass term of the Wilson-like action will mix with the original trace anomaly, since its bare quark mass suffers linear divergence. The Wilson term also leads to a large discrepancy between the renormalization constants of singlet and nonsinglet scalar currents [21]. For a staggered fermion, one needs to overcome the taste mixing problem; the ground-state mass of the baryon is not degenerate. Thus, the overlap fermion is a good choice to evade these difficulties.

The overlap Dirac operator is written as [22-24]

$$
D_{o v}(\rho)=\rho\left(1+\gamma_{5} \epsilon\left(\gamma_{5} D_{w}(-\rho)\right)\right) .
$$

$D_{w}(-\rho)$ is the Wilson fermion operator, while $\rho$ is the mass parameter and is chosen to be $\rho=1.5$ in our calculation. $\epsilon$ is the sign function and satisfies $\epsilon^{2}=1 . D_{o v}(\rho)$ satisfies the Ginsparg-Wilson relation [25]:

$$
D_{o v} \gamma_{5}+\gamma_{5} D_{o v}=\frac{1}{\rho} D_{o v} \gamma_{5} D_{o v} .
$$

The effective Dirac operator can be defined as

$$
D_{c}=\frac{D_{o v}}{1-\frac{1}{2 \rho} D_{o v}} .
$$

$D_{c}$ is chiral and satisfies $\left\{D_{c}, \gamma_{5}\right\}=0$ [26]; this relation also frees the overlap fermion from the flavor mixing of the scalar operator in the massless renormalization schemee.g., the $\overline{\mathrm{MS}}$ scheme. More generally, this mixing effect will occur if one chooses a massive renormalization scheme; even so, one can anticipate that this mixing effect is small, since it occurs in the two-loop level.

In this work, we present the first demonstration of the quantum trace anomaly contribution for three types of hadrons since this mass generation mechanism was proposed more than 40 years ago. We confirm that Eq. (2) is satisfied in all hadrons we calculated in this work, with $\gamma_{m}$ and $\beta$ determined nonperturbatively. In addition, we also find that the gluon trace anomaly density in the pion turns out to be much smaller than that in the other hadrons like nucleons and vector mesons, due to the significant difference in the gluon trace anomaly distribution inside the hadrons.

\section{NUMERICAL SETUP}

We perform the calculation on a $24^{3} \times 642+1$-flavor domain-wall fermion ensemble from the RBC collaboration [27,28], with the pion mass $m_{\pi}=340 \mathrm{MeV}$. The other information of the ensemble we use summarized in Table I.

TABLE I. Information of the RBC ensemble [27,28] used in calculation. The pion and kaon masses are in units of MeV.

\begin{tabular}{lllcccc}
\hline \hline Symbol & $L^{3} \times T$ & $a(\mathrm{fm})$ & $6 / g^{2}$ & $m_{\pi}$ & $m_{K}$ & $N_{\mathrm{cfg}}$ \\
\hline $24 \mathrm{I}$ & $24^{3} \times 64$ & $0.1105(3)$ & 2.13 & $340(1)$ & $593(1)$ & 203 \\
\hline \hline
\end{tabular}


For the valence quark, we use the chiral fermion through the overlap approach to avoid the additional term in the trace of EMT under the lattice regularization.

In order to determine the values of $\gamma_{m}$ and $\beta$ precisely and make an accurate verification of the sum rule, we consider the partially quenched QCD [29], which allows the valence quark mass to be different from that in the gauge ensemble. In such a case, Eq. (2) becomes

$$
M_{H}=\left(1+\gamma_{m}\right)\left(\left\langle H_{m}\right\rangle_{H}^{v}+\left\langle\sum_{i} m_{i} \bar{q}_{i} q_{i}\right\rangle_{H}\right)+\left\langle H_{a}^{g}\right\rangle_{H},
$$

where $H_{a}^{g}=\frac{\beta}{2 g} F^{2},\left\langle H_{m}\right\rangle_{H}^{v}$ includes the connected quark diagrams with the operator $m_{v} \bar{q}_{v} q_{v}$ only, and the index $i$ just includes the sea quark flavors in the gauge configurations: degenerated light up/down and also strange quarks. Their contributions can only be obtained by calculating the disconnected insertion diagrams.

In this work, we consider the cases with five quark masses: $m_{v}=0.026,0.092,0.160,0.319$, and $0.479 \mathrm{GeV}$. Only the lightest quark mass corresponds to the unitary point; the lightest two quark masses represent the light and strange quark masses in the gauge ensemble we used.

First of all, the hadron mass can be extracted from the twopoint correlation function with wall source and wall sink

$$
C_{2}\left(t_{f} ; \mathcal{H}\right)=\left\langle\sum_{\vec{y}} \mathcal{H}\left(t_{f}, \vec{y}\right) \sum_{\vec{x}} \mathcal{H}^{\dagger}(0, \vec{x})\right\rangle,
$$

where $\mathcal{H}$ is the hadron interpolation field. As implemented in Ref. [30], when we calculate the contribution of valence quark mass, we use the Feynman-Hellmann-theoreminspired method [31] to construct the summed three pointcorrelation function for the connected insertion case,

$$
S C_{3}^{q_{v}}\left(t_{f} ; \mathcal{H}\right)=\left\langle\sum_{\vec{y}} \mathcal{H}\left(t_{f}, \vec{y}\right) \sum_{t, \vec{z}} O^{q_{v}}(t, \vec{z}) \sum_{\vec{x}} \mathcal{H}^{\dagger}(0, \vec{x})\right\rangle,
$$

where $O^{q_{v}}=m_{q}^{v} \bar{q}_{v} q_{v}$ is the valence quark operator. For the disconnected insertion case, we modify the expression into

$$
\begin{aligned}
& S C_{3}^{q_{s}, g}\left(t_{f} ; \mathcal{H}\right) \\
& \quad=\left\langle\sum_{\vec{y}} \mathcal{H}\left(t_{f}, \vec{y}\right) \sum_{t \in\left(t_{f}, 0\right), \vec{z}} O^{q_{s}, g}(t, \vec{z}) \sum_{\vec{x}} \mathcal{H}^{\dagger}(0, \vec{x})\right\rangle,
\end{aligned}
$$

where the sum of the current time slices in the light sea quark operator $O_{q}=m_{s} \bar{q}_{s} q_{s}$ (where $m_{s}$ is the sea quark mass) and the gluon operator $O^{g}=F^{2}$ are limited to $t \in\left(t_{f}, 0\right)$ to remove the statistical uncertainty from the region $t<0$ and $t>t_{f}$, which is irrelevant to the matrix elements we want to calculate, and the gluon field tensor $F_{\mu \nu}$ is defined through the standard clover definition. The detailed expressions of $C_{2}$ and $S C_{3}$ and how to construct them with propagators are shown in the Appendix.

For each hadron, we carry out a joint correlated fit of $C_{2}\left(t_{f} ; H\right)$ and $S C_{3}^{q, g}\left(t_{f} ; H\right)$ to extract the $M_{H},\left\langle H_{m}\right\rangle_{H}$, and $\left\langle F^{2}\right\rangle_{H}$ values simultaneously. As mentioned above, $S C_{3}^{q_{v}, q_{s}, g}\left(t_{f} ; H\right)$ is the summed three-point correlation function which has summed the total contribution of the matrix element of the interpolated quark and gluon operator between 0 and $t_{f}$. The fit expression can be written as [30]

$$
\begin{aligned}
S C_{3}^{i=q_{v}, q_{s}, g}\left(t_{f} ; H\right)= & e^{-M_{H} t_{f}}\left(B_{0} t_{f}\left\langle O^{i}\right\rangle_{H}+B_{2}^{i} e^{-\delta m t_{f}}\right. \\
& \left.+B_{3}^{i} t_{f} e^{-\delta m t_{f}}+B_{4}^{i}\right), \\
C_{2}\left(t_{f} ; H\right)= & B_{0} e^{-M_{H} t_{f}}\left(1+B_{1} e^{-\delta m t_{f}}\right),
\end{aligned}
$$

where $\left\langle O^{q_{v}, q_{s}}\right\rangle_{H}=\left\langle H_{m}^{v, s}\right\rangle_{H} ;\left\langle O^{g}\right\rangle_{H}=\left\langle F^{2}\right\rangle_{H}$; the $e^{-\delta m t_{f}}$ terms are introduced to account for the contamination from higher states; and $\delta m, B_{0}, B_{1}$, and $B_{2, \ldots, 4}^{q_{v}, q_{s}, g}$ are free parameters. The contributions from the transition between the ground state and the higher excited state are included in $B_{2, \ldots, 4}^{q_{v}, q_{s}, g}$. The above form is equivalent to extracting the desired quantities in the large- $t_{f}$ limits [30,31]:

$$
\begin{aligned}
M_{H} & =\log \frac{C_{2}\left(t_{f}-1 ; H\right)}{C_{2}\left(t_{f} ; H\right)}+\mathcal{O}\left(e^{-\delta m t_{f}}\right), \\
\left\langle H_{m}\right\rangle_{H} & =\Delta R^{q}\left(t_{f} ; H\right)+\mathcal{O}\left(e^{-\delta m t_{f}}\right), \\
\left\langle F^{2}\right\rangle_{H} & =\Delta R^{g}\left(t_{f} ; H\right)+\mathcal{O}\left(e^{-\delta m t_{f}}\right),
\end{aligned}
$$

where $\Delta R^{i=q, g}\left(t_{f}\right) \equiv \frac{S C_{3}^{i}\left(t_{f} ; H\right)}{C_{2}\left(t_{f} ; H\right)}-\frac{S C_{3}^{i}\left(t_{f}-1 ; H\right)}{C_{2}\left(t_{f}-1 ; H\right)}$. When $t_{f}$ is large enough, the terms proportional to $\mathcal{O}\left(e^{-\delta m t_{f}}\right)$ are negligible, so the three summed functions can be rewritten into the following form:

$$
\begin{aligned}
S C_{3}^{q_{v}, q_{s}, g}\left(t_{f} ; H\right) & =e^{-M_{H} t_{f}}\left(B_{0} t_{f}\left\langle O^{q_{v}, q_{s}, g}\right\rangle_{H}+B_{4}\right), \\
C_{2}\left(t_{f} ; H\right) & =e^{-M_{H} t_{f}} B_{0} .
\end{aligned}
$$

Then, one can obtain that $\left\langle O^{q_{v}, q_{s}, g}\right\rangle_{H}=\frac{S C_{3}^{i}\left(t_{f} ; H\right)}{C_{2}\left(t_{f} ; H\right)}-$ $\frac{S C_{3}^{i}\left(t_{f}-1 ; H\right)}{C_{2}\left(t_{f}-1 ; H\right)}$ in the large- $t_{f}$ limit.

In the actual calculation, we perform the joint fit for the summed three- and two-point functions to extract the matrix element of the ground state. The fit results of $\chi^{2} /$ d.o.f., the mass of the ground-state hadron $M_{H}$, the expectation value of the quark mass $\sum_{q}\left\langle m_{q} \bar{q} q\right\rangle_{H}$, and the gluon operators $-\left\langle F^{2}\right\rangle_{H}$ and $\delta_{m}$ are listed in Table II for all the hadrons used in this work. Note that one needs to substitute $S C_{2,3}\left(t_{f}\right)$ into $S C_{2,3}\left(t_{f}\right)+S C_{2,3}\left(T a-t_{f}\right)$ for the PS meson case in order to include the loop-around effect and describe the data around $t_{f} \sim T a / 2$. 
TABLE II. The $\chi^{2} /$ d.o.f., $M_{H}, \sum_{q}\left\langle m_{q} \bar{q} q\right\rangle_{H},-\left\langle F^{2}\right\rangle_{H}$, and $\delta_{m}$ based on the joint fit for different channels with $m_{v}=0.026,0.092$, $0.160,0.319$, and $0.479 \mathrm{GeV}$.

\begin{tabular}{|c|c|c|c|c|c|c|c|c|c|}
\hline \multirow{2}{*}{$\begin{array}{l}m_{v}(\mathrm{GeV}) \\
\text { Channel }\end{array}$} & \multicolumn{3}{|c|}{0.026} & \multicolumn{3}{|c|}{0.092} & \multicolumn{3}{|c|}{0.160} \\
\hline & PS & $\mathrm{V}$ & $\mathrm{N}$ & PS & V & $\mathrm{N}$ & PS & $\mathrm{V}$ & $\mathrm{N}$ \\
\hline$\chi^{2} /$ d.o.f. & 1.20 & 1.22 & 0.63 & 1.18 & 0.68 & 0.84 & 1.11 & 0.74 & 0.82 \\
\hline$M_{H}(\mathrm{GeV})$ & $0.340(1)$ & $0.881(4)$ & $1.161(6)$ & $0.647(1)$ & $1.027(2)$ & $1.468(4)$ & $0.864(1)$ & $1.174(2)$ & $1.733(3)$ \\
\hline$\sum_{q}\left\langle m_{q} \bar{q} q\right\rangle_{H}(\mathrm{GeV})$ & $0.176(2)$ & $0.126(5)$ & $0.223(14)$ & $0.341(2)$ & $0.254(6)$ & $0.447(10)$ & $0.477(2)$ & $0.387(4)$ & $0.645(9)$ \\
\hline$-\left\langle F^{2}\right\rangle_{H}(\mathrm{GeV})$ & $1.25(42)$ & $9.83(1.45)$ & $10.92(1.48)$ & $1.84(10)$ & $8.84(45)$ & $13.46(1.46)$ & $2.23(12)$ & $8.93(43)$ & $11.77(1.07)$ \\
\hline$\delta m(\mathrm{GeV})$ & $1.07(12)$ & $1.22(17)$ & $0.72(7)$ & $1.00(5)$ & $1.01(10)$ & $0.61(4)$ & $1.02(8)$ & $0.82(5)$ & $0.54(3)$ \\
\hline$m_{v}$ & \multicolumn{5}{|c|}{0.319} & \multicolumn{4}{|c|}{0.479} \\
\hline Channel & \multicolumn{2}{|c|}{ PS } & V & \multicolumn{2}{|c|}{$\mathrm{N}$} & PS & \multicolumn{2}{|r|}{ V } & $\mathrm{N}$ \\
\hline$\chi^{2} /$ d.o.f. & \multicolumn{2}{|c|}{1.11} & 1.06 & \multicolumn{2}{|c|}{0.98} & 0.96 & \multicolumn{2}{|c|}{1.26} & 1.18 \\
\hline$M_{H}(\mathrm{GeV})$ & \multicolumn{2}{|c|}{$1.277(1)$} & $1.505(1)$ & \multicolumn{2}{|c|}{$2.280(2)$} & $1.640(1)$ & \multicolumn{2}{|c|}{$1.825(1)$} & $2.783(2)$ \\
\hline$\sum_{q}\left\langle m_{q} \bar{q} q\right\rangle_{H}(\mathrm{GeV})$ & \multicolumn{2}{|c|}{$0.770(2)$} & $0.682(3)$ & \multicolumn{2}{|c|}{$1.081(8)$} & $1.047(1)$ & \multicolumn{2}{|c|}{$0.953(1)$} & $1.478(1)$ \\
\hline$-\left\langle F^{2}\right\rangle_{H}(\mathrm{GeV})$ & \multicolumn{2}{|c|}{$2.77(11)$} & $7.69(26)$ & \multicolumn{2}{|c|}{$10.31(90)$} & $2.52(28)$ & \multicolumn{2}{|c|}{$6.52(60)$} & $9.56(37)$ \\
\hline$\delta m(\mathrm{GeV})$ & \multicolumn{2}{|c|}{$0.78(4)$} & $0.68(3)$ & \multicolumn{2}{|c|}{$0.48(2)$} & $0.72(1)$ & \multicolumn{2}{|c|}{$0.66(4)$} & $0.44(2)$ \\
\hline
\end{tabular}

\section{RESULT}

In this work, we calculate the quark propagator at five different valence quark masses- $m_{v}=0.026,0.092$, $0.160,0.319$, and $0.479 \mathrm{GeV}$ - and construct the twoand three-point correlation functions for the nucleon $(\mathrm{N})$, pseudoscalar (PS), and vector (V) mesons. To suppress the statistical uncertainty, we apply five steps of the HYP smearing on the gauge operator, repeating the calculation at all 64 time slices and taking the averaged result on each configuration as an independent sample.

In Fig. 1, we plot the effective ratios $\Delta R^{q, g}\left(t_{f}\right)$ defined in Eq. (12) for the PS (blue) and V (red) mesons with $m_{v}=0.479 \mathrm{GeV}$. The disconnected light quark

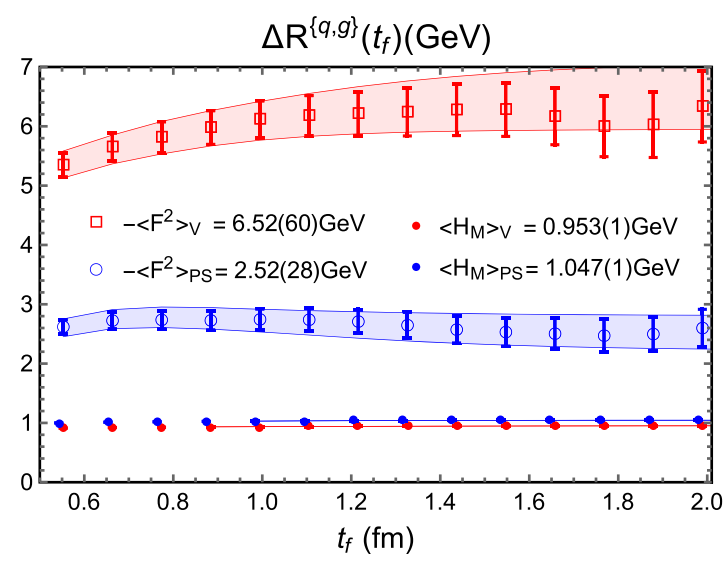

FIG. 1. The differential ratios $\Delta R_{P S, V}^{q, g}\left(t_{f}\right)$ of the pseudoscalar and vector mesons with $m_{v}=0.479 \mathrm{GeV}$, which should approach the ground-state matrix elements $\left\langle H_{m}\right\rangle_{P S, V}$ and $-\left\langle F^{2}\right\rangle_{P S, V}$, respectively, at the $t_{f} \rightarrow \infty$ limit. The bands show the joint fit prediction using the form defined in Eq. (11), and they agree with the data well. contributions are not shown here, as their contributions are small, about $1 \%$. The $M_{H},\left\langle H_{m}\right\rangle_{H}$, and $\left\langle F^{2}\right\rangle_{H}$ values are obtained using the joint fit defined in Eq. (11) with $\chi^{2} /$ d.o.f. $\sim 1$, and the bands of $\Delta R^{q, g}\left(t_{f}\right)$ predicted by the joint fit are also shown in Fig. 1 and agree with the data perfectly. We can see that the gluon trace anomaly matrix element $-\left\langle F^{2}\right\rangle_{H}=-\left.\Delta R^{g}\left(t_{f}\right)\right|_{t_{f} \rightarrow \infty}$ in the $\mathrm{V}$ meson is more than twice that in the PS meson, even though their quark mass terms $\left\langle H_{m}\right\rangle_{H}=\left.\Delta R^{q}\left(t_{f}\right)\right|_{t_{f} \rightarrow \infty}$ only differ from each other by $10 \%$.

Since the anomalous dimension $\gamma_{m}$ and $\beta$ should be independent of the hadron states, we solve the equations

$$
\begin{aligned}
M_{P S}-\left(1+\gamma_{m}\right)\left\langle H_{m}\right\rangle_{P S}-\left.\frac{\beta}{2 g}\left\langle F^{2}\right\rangle_{P S}\right|_{m_{v} a=0.3} & =0, \\
M_{V}-\left(1+\gamma_{m}\right)\left\langle H_{m}\right\rangle_{V}-\left.\frac{\beta}{2 g}\left\langle F^{2}\right\rangle_{V}\right|_{m_{v} a=0.3} & =0
\end{aligned}
$$

and obtain the bare $\gamma_{m}=0.38(3)$ and $\frac{\beta}{2 g}=-0.08(1)$. Both $\gamma_{m}$ and $\frac{\beta}{2 g}$ are small, since they are $\mathcal{O}\left(\alpha_{s}\right)$, so $\left\langle F^{2}\right\rangle_{P S / V}$ should be large enough to satisfy the sum rule.

On the lattice QCD, the ensembles are made up of gauge links instead of gauge fields. A gauge link is a function of the gauge field $A^{\mu}$ multiplied by the coupling constant $g$, and the renormalization of both $g$ and $A^{\mu}$ depend on the discretized gauge faction. On the other hand, $\left\langle g^{2} F^{2}\right\rangle_{H}$ is renormalization independent for the massless QCD at the one-loop level [12]. Hence, it is more natural to consider $\left\langle g^{2} F^{2}\right\rangle_{H}$ on the lattice instead of $\left\langle F^{2}\right\rangle_{H}$. Thus, we divide the coefficient $\frac{\beta}{g}$ by $g^{2}$ and obtain $\frac{\beta}{g^{3}}=-0.056(6)$. Such a value perfectly agrees with the regularization-independent leading order $\frac{\beta^{(0)}}{g^{3}}=\left(-11+\frac{2 N_{f}}{3}\right) /(4 \pi)^{2}=-0.057(7)$, with the 


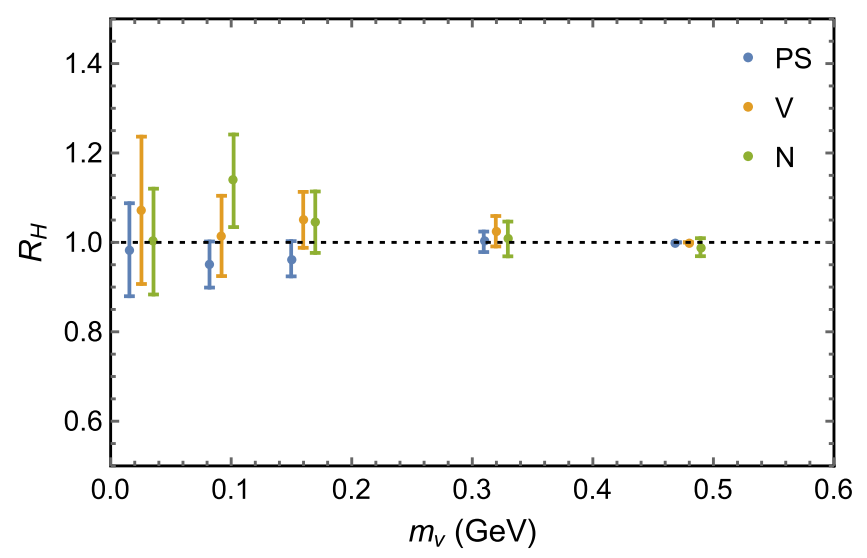

FIG. 2. The ratio of the hadron masses from the left- and righthand sides of Eq. (2), using the $\gamma_{m}$ and $\beta / g$ obtained from the PS and $\mathrm{V}$ mesons with $m_{v}=0.479 \mathrm{GeV}$. It is consistent with 1 within the uncertainties in all the cases.

uncertainty from the next-to-leading-order correction using the bare $\alpha_{s}$. On the other hand, the $\gamma_{m}=0.38(3)$ we obtained is comparable with that under the $\overline{\mathrm{MS}}$ $(1 / a=1.78 \mathrm{GeV})$, which is $0.325(10)$ [32], with the error estimated from the $\mathcal{O}\left(\alpha_{s}^{4}\right)$ correction.

With the above values of $\gamma_{m}$ and $\frac{\beta}{2 g}$, we calculate the hadron mass $M_{H}$, quark mass term $\left\langle H_{m}\right\rangle_{H}$, and $\left\langle F^{2}\right\rangle_{H}$ of the pseudoscalar, vector meson, and nucleon with different $m_{v}$ 's, and plot the ratio

$$
R_{H}\left(m_{v}\right)=\frac{\left(1+\gamma_{m}\right)\left\langle H_{m}\right\rangle_{H}+\frac{\beta}{2 g}\left\langle F^{2}\right\rangle_{H}}{M_{H}}
$$

in Fig. 2. We can see that the $R_{H}$ in all the cases is consistent with 1 within the uncertainties. Note that the PS and $\mathrm{V}$ meson cases with $m_{v}=0.479 \mathrm{GeV}$ are exactly 1 , since they are the input cases. Such a result verifies the trace anomaly sum rule in Eq. (2), and is consistent with our expectation that both $\gamma_{m}$ and $\beta$ are universal.

The resulting gluonic trace anomaly contribution $\left\langle H_{a}^{g}\right\rangle_{H}=\left\langle\frac{\beta}{2 g} F^{2}\right\rangle_{H}$ in the $2+1$ flavor ensemble are plotted in Fig. 3. We can see that $\left\langle H_{a}^{g}\right\rangle_{H}$ in the pion state is generally smaller than in the other states, especially at the unitary point for the $340 \mathrm{MeV}$ pion mass. In such a case, the gluon trace anomaly contributes about $100 \mathrm{MeV}$ of the pion mass, which is $\sim 30 \%$, but $\sim 800 \mathrm{MeV}$ in the $\rho$ meson and nucleon cases. It is exactly what we expected from the trace anomaly sum rule: the trace anomaly contributes most of the hadron masses, except for the pion case.

The difference will be much more significant at the physical quark mass. If we use the GMOR relation $M_{\pi}^{2} \propto m_{q}$ and the Feynman-Hellman theorem $\left\langle H_{m}\right\rangle_{H}=m_{q} \frac{\partial M_{\pi}}{\partial m_{q}}=$ $\frac{1}{2} M_{\pi}+\mathcal{O}\left(M_{\pi}^{3}\right)$ [7,33], we can estimate the gluon trace anomaly contribution in the physical pion state to be

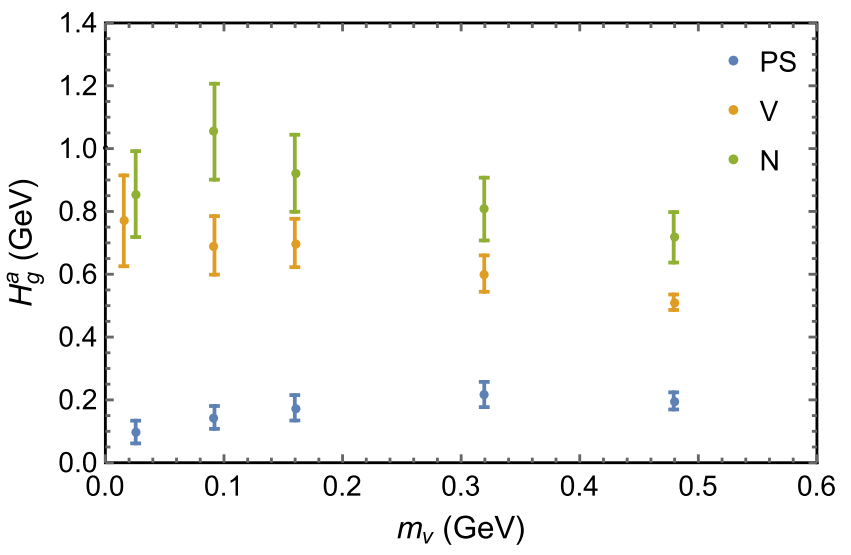

FIG. 3. The gluon trace anomaly contribution to the hadron mass. For five different quark masses, the corresponding pion masses are $0.340,0.647,0.864,1.277$, and $1.640 \mathrm{GeV}$. We can see that it is always small for the PS meson, while it approaches $\sim 800 \mathrm{MeV}$ for the nucleon and vector mesons in the chiral limit $m_{v} \rightarrow 0$.

$\frac{1}{2}\left(1-\gamma_{m}\right) M_{\pi}=43(2) \mathrm{MeV}$. On the other hand, the contribution in the nucleon at the physical light quark mass will be $816(10) \mathrm{MeV}$ if we only consider the quark mass contribution from three light quarks $[10,11]$.

In order to uncover the origin of this difference, we also investigate the gluon trace anomaly density inside the hadron. Similarly to the previous study about the pion charged radius [34], the density of the gluon trace anomaly can be defined as

$\rho_{H}(|r|)=\left.\frac{\left\langle\sum_{\vec{y}} \mathcal{H}\left(t_{f}, \vec{y}\right) H_{a}^{g}(t, \vec{y}+\vec{r}) \sum_{\vec{x}} \mathcal{H}^{\dagger}(0, \vec{x})\right\rangle}{\left\langle\sum_{\vec{y}} \mathcal{H}\left(t_{f}, \vec{y}\right) \sum_{\vec{x}} \mathcal{H}^{\dagger}(0, \vec{x})\right\rangle}\right|_{t, t_{f}-t \rightarrow \infty}$,

as $\rho_{H}(|r|)$ can be related to the gluon trace anomaly matrix element $\left\langle H_{a}^{g}\right\rangle_{H}=\int_{\vec{r}} \mathrm{~d}^{3} r \rho_{H}(|r|)$ and its squared charge radius $\left\langle r_{g}^{2}\right\rangle_{H}=\frac{\int_{\vec{r}} \mathrm{~d}^{3} r r^{2} \rho_{H}(|r|)}{\left\langle H_{a}^{g}\right\rangle_{H}}$. A naive guess is that $\rho_{H}$ would be insensitive to the hadron at small $r$, while it should decay much faster in the pion than the other hadrons to cause the integral and charge radius to be significantly smaller. But our lattice QCD calculation has excluded such a possibility, as shown in Fig. 4. First of all, the magnitude of $\rho_{H}$ in the nucleon and vector meson is comparable with the leadingorder estimate of the vacuum expectation value $\langle\rho\rangle=$ $-0.007 \mathrm{GeV}^{4}$ [35] at small $r$, even though their signs are opposite. At the same time, one can see that the density of the pion is much smaller than that of the nucleon and the vector meson; even more, the density tends to be negative and then has the same sign as that in the vacuum. It means that $\left\langle H_{a}^{g}\right\rangle$ receives both suppression from the magnitude of density and also short-range interaction, and it can cause the pion-gluon trace anomaly radius to be larger than the 


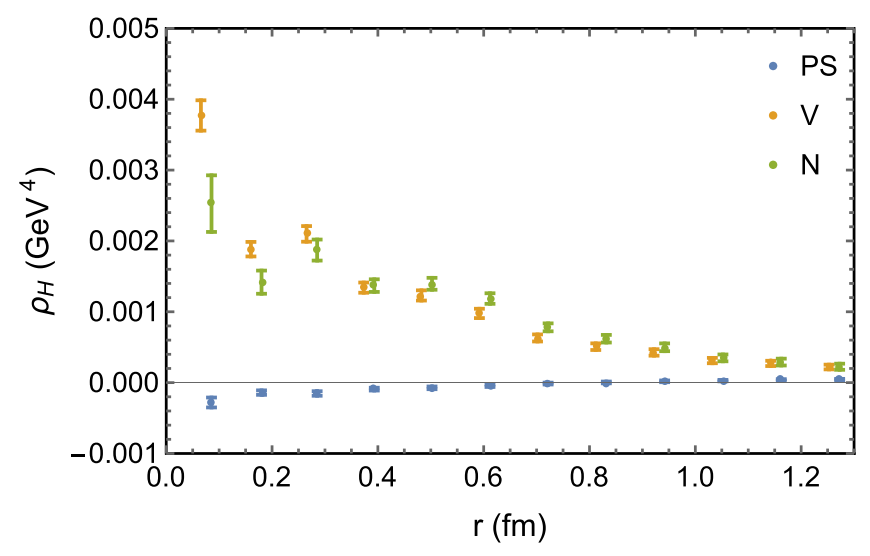

FIG. 4. The density of the gluon trace anomaly in different hadron states at the unitary point for the $340 \mathrm{MeV}$ pion mass.

other hadrons. More details of distribution calculation can be found in the Appendix.

\section{SUMMARY AND OUTLOOK}

In this work, we calculated the quark mass term $\langle m \bar{\psi} \gamma \psi\rangle_{H}$ and gluon action terms $\left\langle F^{2}\right\rangle_{H}$ in the hadron. Based on the EMT trace anomaly sum rule in the PS and V meson states with $m_{v}=0.479 \mathrm{GeV}$, we determined the bare anomalous dimensions of the quark mass and gluon coupling constant as $\gamma_{m}=0.38(3)$ and $\frac{\beta}{g^{3}}=-0.056(6)$ (with five steps of HYP smearing on the gluon operator), respectively, and confirmed that they are independent on hadron states and quark mass up to the statistical uncertainties. With such $\gamma_{m}$ and $\frac{\beta}{g^{3}}$ values, we find that the gluon trace anomaly contribution in the PS meson mass is always much smaller than that in the other hadrons, especially around the chiral limit.

An important check on the trace anomaly mechanism can be carried out by renormalizing the $\left\langle F^{2}\right\rangle_{H}$ nonperturbatively [36] and converting it to that under the $\overline{\mathrm{MS}}$ scheme; then, one can obtain the values of $\gamma_{m}$ and $\frac{\beta}{2 g}$ in the $\overline{\mathrm{MS}}$ scheme and compare them with perturbative results, and/or deduce the conserved EMT with an $\mathcal{O}\left(a^{2}\right)$ trace term directly from the discretized QCD action. The calculations at different lattice spacings, and at the same lattice spacing but with different gauge actions (the Symanzik action used by the MILC configurations and the Iwasaki action used here can cause the bare $g^{2}$ to be different by a factor of 1.7 at $a \sim 0.1 \mathrm{fm})$ are also desirable.

At the same time, it suggests that the coupling between hadrons and the bilinear heavy quark operator can be obtained indirectly based on the relation in Eq. (3). For example, the total trace anomaly contribution including both the $\gamma_{m}$ and $\frac{\beta}{2 g}$ terms are generally $\sim 800 \mathrm{MeV}$ for most hadrons except for light pseudoscalar mesons. But with $\gamma_{m} \sim 0.3$, we will have $\left\langle\frac{\beta}{2 g} F^{2}\right\rangle_{N} \sim 0.84 \mathrm{GeV}$ and $\left\langle\frac{\beta}{2 g} F^{2}\right\rangle_{\eta_{c}} \sim 0.12 \mathrm{GeV}$. And then $\left\langle m_{b} \bar{\psi}_{b} \psi_{b}\right\rangle_{N}$ will be larger than $\left\langle m_{b} \bar{\psi}_{b} \psi_{b}\right\rangle_{\eta_{c}}$ by a factor of $\sim 5$ and can be verified by direct calculations.

Another interesting thing is that the gluon trace anomaly densities in the pion and nucleon are comparable in their extent, while their magnitudes are very different. Moreover, the density in the center of the pion is negative, which is opposite to the density in the nucleon but has the same sign as that in the vacuum. A negative density in the center of the pion is inconsistent with the naive expectation: $\rho_{H}$ at the center would be insensitive to the hadron. It opens a new gate to understanding the relation between pion and vacuum, and why the other hadrons are different from them.

\section{ACKNOWLEDGMENTS}

We thank Heng-Tong Ding, Xiangdong Ji, Luchang Jin, Keh-Fei Liu, Jianhui Zhang, and Jian Zhou for valuable discussions, and the RBC and UKQCD Collaborations for providing us their DWF gauge configurations. The calculations were performed using the GWU code $[37,38]$ through the HIP programming model [39]. The numerical calculation has mainly been done on CAS Xiaodao-1 computing environment, and supported by the Strategic Priority Research Program of Chinese Academy of Sciences, Grant No. XDC01040100, and also the HPC Cluster of Institute of Theoretical Physics, Chinese Academy of Sciences, Jiangsu Key Lab for Jiangsu Key Laboratory for Numerical Simulation of Large Scale Complex Systems. P. S. is supported by the Natural Science Foundation of China under Grant No. 11975127, as well as the Jiangsu Specially Appointed Professor Program. Y. Y. is supported by the Strategic Priority Research Program of Chinese Academy of Sciences, Grants No. XDB34030303, No. XDC01040100, and No. XDPB15. P. S. and Y. Y. are also supported in part by a NSFC-DFG joint grant under Grant No. 12061131006 and SCHA 458/22.

\section{APPENDIX: SUMMARY OF TECHNICAL DETAILS}

\section{Contraction of the Green's functions}

In this section, we will give the expressions of two-point and summed three-point correlation functions constructed by propagator. In our calculation, we use the Coulomb gauge fixed-wall-source propagator $S_{w}\left(\vec{y}, t_{2} ; t_{1}\right)=$ $\sum_{\vec{x}} S\left(\vec{y}, t_{2} ; \vec{x}, t_{1}\right)$ and the Feynman-Hellmann propagator [31] $\tilde{S}_{c}\left(\vec{y}, t_{2} ; t_{1}\right)=m_{q} \sum_{\vec{x}, t} S\left(\vec{y}, t_{2} ; \vec{x}, t\right) S_{w}\left(\vec{x}, t ; t_{1}\right)$ to construct the two-point and connected three-point correlation functions,

$$
\begin{aligned}
C_{2}\left(t_{f} ; \mathcal{M}_{\Gamma}\right) & =\sum_{\vec{y}} C_{2}\left(t_{f}, \vec{y} ; \mathcal{M}_{\Gamma}\right)=C_{\mathcal{M}_{\Gamma}}\left(S_{w}, S_{w}, t_{f}, 0\right), \\
S C_{3}^{q_{v}}\left(t_{f} ; \mathcal{M}_{\Gamma}\right) & =C_{\mathcal{M}_{\Gamma}}\left(S_{c}, S_{w}, t_{f}, 0\right)+C_{\mathcal{M}_{\Gamma}}\left(S_{w}, S_{c}, t_{f}, 0\right),
\end{aligned}
$$


where $C_{\mathcal{M}_{\Gamma}}$ is defined as

$$
C_{\mathcal{M}_{\Gamma}}\left(S_{1}, S_{2}, t_{f}, 0\right)=\sum_{\vec{y}}\left\langle\operatorname{Tr}\left(\gamma_{5} S_{1}^{\dagger}\left(\vec{y}, t_{f} ; 0\right) \gamma_{5} \Gamma S_{2}\left(\vec{y}, t_{f} ; 0\right) \Gamma\right)\right\rangle
$$

For the meson $\mathcal{M}_{\Gamma}$ with the interpolation field $\bar{\psi} \Gamma \psi, S\left(\vec{y}, t_{2} ; \vec{x}, t_{1}\right)$ is the quark propagator from $\left(\vec{x}, t_{1}\right)$ to $\left(\vec{y}, t_{2}\right)$. For the quark loop and gluon operator, we need to calculate the following disconnected three-point correlation functions:

$$
S C_{3}^{q_{s}, g}\left(t_{f}, \mathcal{M}\right)=\sum_{0<t<t_{f}} \sum_{\vec{y}} \sum_{\vec{x}}\left\langle\left[C_{2}\left(t_{f}, \vec{y} ; \mathcal{M}_{\Gamma}\right)-\left\langle C_{2}\left(t_{f}, \vec{y} ; \mathcal{M}_{\Gamma}\right)\right\rangle\right]\left[\mathcal{O}^{q_{s}, g}(t, \vec{x})-\left\langle\mathcal{O}^{q_{s}, g}(t, \vec{x})\right\rangle\right]\right\rangle
$$

The definition of $\mathcal{O}^{q s}$ and $\mathcal{O}^{g}$ is in the following content of Eq. (10). The nucleon case with the interpolation field $\left(u^{T} \tilde{C} d\right) u$ is similar and can be obtained without much modification:

$$
\begin{aligned}
C_{2}\left(t_{f} ; \mathcal{N}\right) & =\sum_{\vec{y}} C_{2}\left(t_{f}, \vec{y} ; \mathcal{N}\right)=C_{\mathcal{N}}\left(S_{w}, S_{w}, S_{w}, t_{f}, 0\right), \\
S C_{3}^{q_{v}}\left(t_{f} ; \mathcal{N}\right)= & C_{\mathcal{N}}\left(S_{c}, S_{w}, S_{w}, t_{f}, 0\right)+C_{\mathcal{N}}\left(S_{w}, S_{c}, S_{w}, t_{f}, 0\right)+C_{\mathcal{N}}\left(S_{w}, S_{w}, S_{c}, t_{f}, 0\right), \\
C_{\mathcal{N}}\left(S_{1}, S_{2}, S_{3}, t_{2}, t_{1}\right)= & \left\langle\epsilon ^ { a b c } \epsilon ^ { a ^ { \prime } b ^ { \prime } c ^ { \prime } } \sum _ { \vec { y } } \operatorname { T r } ( \Gamma _ { m } S _ { 1 } ^ { a a ^ { \prime } } ( \vec { y } , t _ { f } ; 0 ) ) \operatorname { T r } \left(\underline{S}_{2}^{b b^{\prime}}\left(\left(\vec{y}, t_{f} ; 0\right) S_{3}^{c c^{\prime}}\left(\vec{y}, t_{f} ; 0\right)\right)\right.\right. \\
& \left.+\operatorname{Tr}\left(\Gamma_{m} S_{1}^{a a^{\prime}}\left(\vec{y}, t_{f} ; 0\right) \underline{S}_{2}^{b b^{\prime}}\left(\vec{y}, t_{f} ; 0\right) S_{3}^{c c^{\prime}}\left(\vec{y}, t_{f} ; 0\right)\right)\right\rangle,
\end{aligned}
$$

where $\underline{S}$ is defined by $\left(\tilde{C} S \tilde{C}^{-1}\right)^{T}, \tilde{C}=\gamma_{2} \gamma_{4} \gamma_{5}$, and $\Gamma_{m}=\frac{1}{2}\left(1+\gamma_{4}\right)$ is the unpolarized projector. The summed three-point correlation functions for a light quark loop and gluon are defined as

$$
S C_{3}^{q_{s}, g}\left(t_{f} ; \mathcal{N}\right)=\sum_{0<t<t_{f}} \sum_{\vec{y}} \sum_{\vec{x}}\left\langle\left[C_{2}\left(t_{f}, \vec{y} ; \mathcal{N}\right)-\left\langle C_{2}\left(t_{f}, \vec{y} ; \mathcal{N}\right)\right\rangle\right]\left[\mathcal{O}^{q_{s}, g}(t, \vec{x})-\left\langle\mathcal{O}^{q_{s}, g}(t, \vec{x})\right\rangle\right]\right\rangle .
$$

Note that during the calculation with the overlap fermion, deflating the long-distance subspace of the Dirac operator using its eigenvectors $v(\lambda)$ with $|\lambda|<\Lambda_{\mathrm{QCD}}$ are essential to obtain the light quark propagator efficiently [40]. At the same time, we can build the light quark loop operator $O_{q}(t)$ only via those eigenvectors $v(\lambda)$ with little cost, and the systematic uncertainty from the rest is negligible [10]:

$$
\begin{aligned}
O_{q}(t) & \equiv \sum_{\vec{x}} S\left(m_{q} ; \vec{x}, t ; \vec{x}, t\right) \simeq \sum_{\vec{x}} S^{L}\left(m_{q} ; \vec{x}, t ; \vec{x}, t\right), \\
S^{L}\left(m_{q}\right) & \equiv \int_{-i \Lambda_{\mathrm{QCD}}}^{i \Lambda_{\mathrm{QCD}}} \mathrm{d} \lambda \frac{v(\lambda) v^{\dagger}(\lambda)}{\lambda+m_{q}} .
\end{aligned}
$$

In practice, 300 pairs of the low-lying eigenpairs of the Dirac operator with $\lambda \leq 0.246 \mathrm{GeV}$ are solved to speed up the propagator calculation and construct the light quark loops.

The clover definition of $F_{\mu \nu}$ used for the gluon operator $F^{2}$ is the following:

$$
\begin{aligned}
g F_{\mu \nu}(x) & =\frac{i}{8 a^{2}}\left[\mathcal{P}_{[\mu, \nu]}+\mathcal{P}_{[\nu,-\mu]}+\mathcal{P}_{[-\mu,-\nu]}+\mathcal{P}_{[-\nu, \mu]}\right](x), \\
\mathcal{P}_{\mu, \nu}(x) & =U_{\mu}(x) U_{\nu}(x+a \hat{\mu}) U_{\mu}^{\dagger}(x+a \hat{\nu}) U_{\nu}^{\dagger}(x),
\end{aligned}
$$

where $U_{-\nu}(x)=U_{\nu}^{\dagger}(x-a \hat{\nu})$ and $P_{[\mu, \nu]} \equiv P_{\mu, \nu}-P_{\nu, \mu}$.

\section{The trace anomaly density and radius in the hadrons}

We calculate the trace anomaly density using the following summed three-point correlation function,

$$
S C_{3}^{g}\left(t_{f}, r ; \mathcal{H}\right)=\sum_{0<t<t_{f}} \sum_{\vec{x}} \sum_{|\vec{y}-\vec{x}|=r}\left\langle\left[\left\langle C_{2}\left(t_{f}, \vec{x} ; \mathcal{H}\right)-\left\langle C_{2}\left(t_{f}, \vec{x} ; \mathcal{H}\right)\right\rangle\right\rangle\right]\left[F^{2}(\vec{y}, t)-\frac{1}{V}\left\langle\sum_{\vec{z}} F^{2}(\vec{z}, t)\right\rangle\right]\right\rangle
$$

and then $\rho_{H}$ can be obtained by

$$
\rho_{H}(r)=\left.\frac{\beta}{2 g \sum_{|\vec{y}-\vec{x}|=r} 1}\left(\frac{S C_{3}^{g}\left(t_{f}, r ; \mathcal{H}\right)}{C_{2}\left(t_{f} ; \mathcal{H}\right)}-\frac{S C_{3}^{g}\left(t_{f}-1, r, \mathcal{H}\right)}{C_{2}\left(t_{f}-1, \mathcal{H}\right)}\right)\right|_{t_{f} \rightarrow \infty, L \rightarrow \infty}
$$


In practice, we use the joint fit defined in Eqs. (8) and (9) to eliminate the excited-state contamination with the data at finite $t_{f}$.

Generally, the charge radius can be defined through the integration of the normalized density $\bar{\rho}_{H}(r)=\frac{\rho_{H}(r)}{\int \mathrm{d}^{3} r^{\prime \prime} \rho_{H}\left(r^{\prime \prime}\right)}$,

$$
\left\langle r_{g}^{2}\right\rangle_{H}=\int \mathrm{d}^{3} r^{\prime} r^{2} \bar{\rho}_{H}\left(r^{\prime}\right)
$$

But in a finite volume $L^{3} \times T$, such a definition can only be accurate when $\bar{\rho}_{H}(r)$ is negligible at $r \sim L / 2$; otherwise, it will receive additional contributions from $\rho_{H}(L-r)$. Thus, we access the charge radius through the form factor $F_{g}\left(Q^{2}\right)$ as a function of the momentum transfer $Q^{2}$,

$$
\left\langle r_{g}^{2}\right\rangle_{H}=\left.\frac{6}{F_{g}(0)} \frac{\mathrm{d} F_{g}\left(Q^{2}\right)}{\mathrm{d} Q^{2}}\right|_{Q^{2} \rightarrow 0},
$$

which is a more effective way to reduce the containment from the region $r>L / 2$. A similar calculation at larger size is used to suppress the finite volume effect.

As shown in Fig. 5, $\bar{\rho}_{H}$ is still nonzero at $r=L / 2$, especially for the pion case. Thus, integrating it with $r^{2}$ to obtain the charge radius can have huge systematic uncertainty. But as in the figure, $\bar{\rho}_{\pi}(r)$ is smaller than $\bar{\rho}_{N}(r)$ for all the $r<1.1 \mathrm{fm}$. Thus, generally, we can rewrite the pion charge radius into

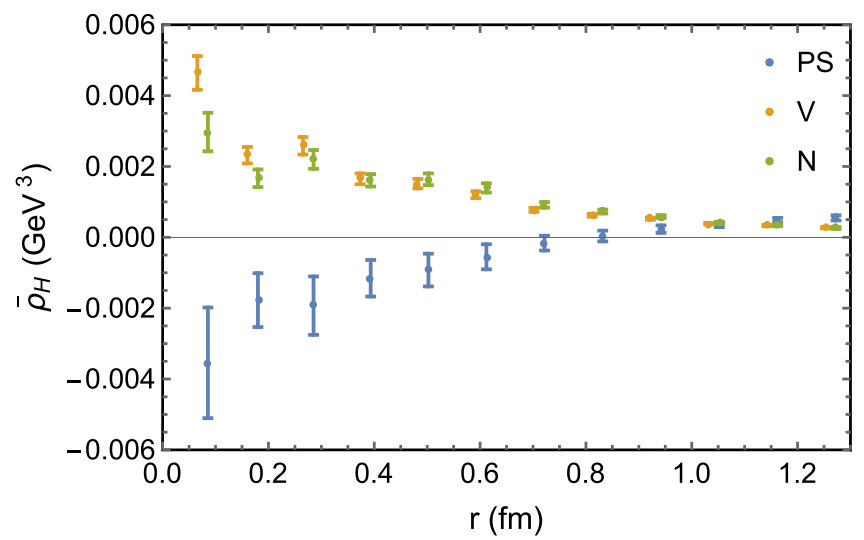

FIG. 5. The normalized density of the gluon trace anomaly in the different hadron states at the unitary point for a $340 \mathrm{MeV}$ pion mass.

$$
\begin{aligned}
\left\langle r_{g}^{2}\right\rangle_{\pi}= & \int \mathrm{d}^{3} r^{\prime} r^{\prime 2} \bar{\rho}_{\pi}\left(r^{\prime}\right)=\left\langle r_{g}^{2}\right\rangle_{N} \\
& +\int \mathrm{d}^{3} r^{\prime}\left(r^{\prime 2}-r_{0}^{2}\right)\left(\bar{\rho}_{\pi}\left(r^{\prime}\right)-\bar{\rho}_{N}\left(r^{\prime}\right)\right),
\end{aligned}
$$

where $r_{0}$ is the intersection of $\bar{\rho}_{\pi}\left(r_{0}\right)$ and $\bar{\rho}_{N}\left(r_{0}\right)$, and we have $\bar{\rho}_{\pi}\left(r_{0}\right)=\bar{\rho}_{N}\left(r_{0}\right)$ at $r_{0}$. Then the integrand of the second term on the right-hand side will be always positive, and one can expect $\left\langle r_{g}^{2}\right\rangle_{\pi}>\left\langle r_{g}^{2}\right\rangle_{N}$.
[1] Y. Hatta, A. Rajan, and K. Tanaka, J. High Energy Phys. 12 (2018) 008.

[2] S. Caracciolo, G. Curci, P. Menotti, and A. Pelissetto, Nucl. Phys. B309, 612 (1988).

[3] S. Caracciolo, P. Menotti, and A. Pelissetto, Nucl. Phys. B375, 195 (1992).

[4] J. C. Collins, A. Duncan, and S. D. Joglekar, Phys. Rev. D 16, 438 (1977).

[5] N. K. Nielsen, Nucl. Phys. B120, 212 (1977).

[6] M. A. Shifman, A. I. Vainshtein, and V. I. Zakharov, Phys. Lett. 78B, 443 (1978).

[7] X.-D. Ji, Phys. Rev. D 52, 271 (1995).

[8] X. Ji and Y. Liu, Sci. China Phys. Mech. Astron. 64, 281012 (2021).

[9] B.-D. Sun, Z.-H. Sun, and J. Zhou, Phys. Rev. D 104, 056008 (2021).

[10] Y.-B. Yang, A. Alexandru, T. Draper, J. Liang, and K.-F. Liu (xQCD Collaboration), Phys. Rev. D 94, 054503 (2016).

[11] Y.-B. Yang, J. Liang, Y.-J. Bi, Y. Chen, T. Draper, K.-F. Liu, and Z. Liu, Phys. Rev. Lett. 121, 212001 (2018).

[12] R. Tarrach, Nucl. Phys. B196, 45 (1982).

[13] A. Accardi et al., Eur. Phys. J. A 52, 268 (2016).

[14] D. P. Anderle et al., Front. Phys. (Beijing) 16, 64701 (2021).
[15] M. E. Luke, A. V. Manohar, and M. J. Savage, Phys. Lett. B 288, 355 (1992).

[16] D. Kharzeev, Proc. Int. Sch. Phys. Fermi 130, 105 (1996).

[17] D. Kharzeev, H. Satz, A. Syamtomov, and G. Zinovjev, Eur. Phys. J. C 9, 459 (1999).

[18] R. Boussarie and Y. Hatta, Phys. Rev. D 101, 114004 (2020).

[19] P. Sun, X.-B. Tong, and F. Yuan, arXiv:2103.12047.

[20] Y. Guo, X. Ji, and Y. Liu, Phys. Rev. D 103, 096010 (2021).

[21] G. S. Bali, S. Collins, D. Richtmann, A. Schäfer, W. Söldner, and A. Sternbeck (RQCD Collaboration), Phys. Rev. D 93, 094504 (2016).

[22] R. Narayanan and H. Neuberger, Nucl. Phys. B443, 305 (1995).

[23] H. Neuberger, Phys. Lett. B 417, 141 (1998).

[24] K.-F. Liu, Int. J. Mod. Phys. A 20, 7241 (2005).

[25] P. H. Ginsparg and K. G. Wilson, Phys. Rev. D 25, 2649 (1982).

[26] T.-W. Chiu and S. V. Zenkin, Phys. Rev. D 59, 074501 (1999).

[27] T. Blum et al. (RBC, UKQCD Collaborations), Phys. Rev. D 93, 074505 (2016).

[28] R. D. Mawhinney (RBC, UKQCD Collaboration), arXiv: 1912.13150. 
[29] C. W. Bernard and M. F. L. Golterman, Phys. Rev. D 49, 486 (1994).

[30] W. Sun, Y. Chen, P. Sun, and Y.-B. Yang (QCD Collaboration), Phys. Rev. D 103, 094503 (2021).

[31] C. C. Chang, A. N. Nicholson, E. Rinaldi, E. Berkowitz, N. Garron, D. A. Brantley, H. Monge-Camacho, C. J. Monahan, C. Bouchard, M. A. Clark et al., Nature (London) 558, 91 (2018).

[32] K. G. Chetyrkin and A. Retey, Nucl. Phys. B583, 3 (2000).

[33] J. Gasser and H. Leutwyler, Phys. Rep. 87, 77 (1982).

[34] X. Feng, Y. Fu, and L.-C. Jin, Phys. Rev. D 101, 051502 (2020).

[35] M. A. Shifman, A. I. Vainshtein, and V. I. Zakharov, Nucl. Phys. B147, 448 (1979).
[36] Y.-B. Yang, M. Gong, J. Liang, H.-W. Lin, K.-F. Liu, D. Pefkou, and P. Shanahan, Phys. Rev. D 98, 074506 (2018).

[37] A. Alexandru, C. Pelissier, B. Gamari, and F. Lee, J. Comput. Phys. 231, 1866 (2012).

[38] A. Alexandru, M. Lujan, C. Pelissier, B. Gamari, and F. X. Lee, Efficient Implementation of the Overlap Operator on Multi-GPUs (IEEE, 2011), pp. 123-130, https://doi.org/ 10.1109/SAAHPC.2011.13.

[39] Y.-J. Bi, Y. Xiao, M. Gong, W.-Y. Guo, P. Sun, S. Xu, and Y.-B. Yang, Proc. Sci., LATTICE2019 (2020) 286 [arXiv:2001.05706].

[40] A. Li et al. ( $\chi$ QCD Collaboration), Phys. Rev. D 82, 114501 (2010). 